\title{
Análisis de indicadores económicos de turismo para la provincia de Pastaza enfocados al observatorio turístico "UEA"
}

\author{
Analysis of economic indicators of tourism for the province of Pastaza \\ focused on the tourism observatory
}

Cintia del Pilar Hernández Espinosa. ${ }^{1}$, Tania Cristina Cevallos Punguil. ${ }^{2}$, Jaimar Andrea Cifuentes Caiza. ${ }^{3} \&$ Odra Carolina Guerrero Escalante. ${ }^{4}$

\begin{abstract}
.
This research is focused on the Tourism Observatory of the Amazon State University, located in the city of Puyo, Pastaza province.The dynamizing effect that tourism projects on the economy is of notable importance, within the study the impact of tourism on the economic factor is included with the theoretical bases of the tourism system diagramming its development method in a globalized economy that synthesizes the true importance of tourism, by means of a qualitative methodology with a positivist approach for an analyticaldescriptive study, which promotes informative data object of growth and provision of timely information on different tourist areas; The adequate measurement of statistical tourism data are essential tools to help in planning and understanding processes, allowing the adoption of strategies with more possibilities of success in the tourism industry to increase its competitiveness and guarantee economic sustainability.
\end{abstract}

Keywords: Tourism, Economy, Indicators, Tourism Observatory.

\footnotetext{
${ }^{1}$ Universidad Estatal Amazónica, Centro de Posgrados, Maestrante en Turismo, Mención Gestión del Turismo, Puyo-Ecuador, cd.hernandeze@uea.edu.ec

${ }^{2}$ Universidad Estatal Amazónica, Facultad Ciencias de la Vida, Carrera de Turismo, Puyo-Ecuador, tc.cevallosp@uea.edu.ec

${ }^{3}$ Universidad Estatal Amazónica, Centro de Posgrados, Maestrante en Turismo, Mención Gestión del Turismo, Puyo-Ecuador, ja.cifuentesc@uea.edu.ec

${ }^{4}$ Instituto Superior Tecnológico Tena, Docente de la carrera de Turismo, Tena-Ecuador, ocarog21@hotmail.com
} 


\section{Resumen.}

La presente investigación se enfoca en el Observatorio Turístico de la Universidad Estatal Amazónica, localizado en la ciudad de Puyo, provincia de Pastaza. El efecto dinamizador que proyecta el turismo sobre la economía es de notable importancia, dentro del estudio se incluye el impacto del turismo sobre el factor económico con las bases teóricas del sistema turístico diagramando su método de desarrollo en una economía globalizada que sintetiza la verdadera importancia del turismo, mediante una metodología cualitativa con un enfoque positivista para un estudio analítico-descriptivo, que promueve datos informativos objeto de crecimiento y dotación de información oportuna sobre diferentes ámbitos turísticos; la adecuada medición de datos turísticos estadísticos son imprescindibles herramientas para ayudar en la planificación y entender procesos permitiendo adoptar estrategias con más posibilidades de éxito en la industria turística para incrementar su competitividad y garantizar sostenibilidad económica.

Palabras claves: Turismo, Economía, Indicadores, Observatorio Turístico.

\section{Introducción.}

El turismo es considerado un fenómeno económico global que emerge de la necesidad de viajar empleando el tiempo libre o de ocio para destinarlo en actividades de índole turísticas que engloban movimientos económicos dinamizadores capaces de presentar grandes avances mundiales en materia económica, como lo menciona la Organización Mundial del Turismo (OMT) en el último trimestre del año 2018 mediante un análisis sobre el Turismo como líder entre los principales sectores económicos del mundo basado en su barómetro de turismo mientras que el 2019 se fijó al turismo como un importante diversificador con capacidad para ser un motor económico sostenible y de desarrollo en un comunicado de prensa y en septiembre del 2020 en una nota informativa el turismo y el COVID-19 indica que se debe aprovechar el turismo interno para impulsar la recuperación económica.

De acuerdo con lo anteriormente expuesto seleccionar al turismo como uno de los principales actores de la economía no parte de la causalidad, en el año 2019 el Ecuador obtuvo el 1,8\% del Producto Interno Bruto (PIB) mientras que el año 2018 fue del 1,7\% el PIB por concepto de turismo según datos del Banco Central del Ecuador en el mismo periodo para los dos años (Ministerio de Turismo, 2019), es decir el turismo creció de acuerdo con el análisis del indicador económico.

Por ende, el turismo ha generado un crecimiento económico a nivel mundial y nacional junto con el desarrollo de nuevos emprendimientos que originan fuentes de empleo permitiendo el desarrollo de la actividad turística focalizada en la demanda y oferta, haciendo imprescindible tomar alternativas para medir el eficiente uso de los recursos; partiendo del turismo como factor determinante en el estudio de análisis de indicadores económicos. 
Cabe señalar que el turismo desde una óptica económica mantiene una estrecha conexión con un sin número de actividades económicas de consumo corriente (Vogeler y Hernández, 2018, pág. 213), desde este punto de vista es pertinente mencionar que el turismo se apoya en variantes estratégicas de los lugares en los cuales se desarrolla y su consumo no es unicamente demandada por turistas sino también por residentes.

Es importante mencionar que la OMT en el año 2019 en su panel de datos de turismo facilita estadísticas además de datos sobre indicadores clave que muestran al turismo receptor y emisor en una escala regional, nacional y mundial además se encuentra de manera especial dos módulos sobre el impacto que ha tenido el COVID-19 en el turismo. Por lo tanto, para que el turismo posea un adecuado y contundente desarrollo económico requiere de una medición apropiada que faculte la toma de decisiones mediante el uso de indicadores económicos de turismo que cuantifique la eficacia de la actividad o en su defecto sus procesos (Heredia, 2000, pág. 61).

A lo anteriormente expuesto se suma la importante y adecuada participación de Observatorio de Turismo (OT) para la gestión de información y generación de datos pertinentes para la toma de decisiones acertadas en su momento, por tan razón el Sistema Económico Latinoamericano y del Caribe (SELA, 2006, s/p) a quien cita (Angulo, 2009, pág. 9) entre sus principales conclusiones sobres los OT se destaca lo siguiente:

- Los observatorios están destinados a captar, organizar, evaluar y procesar información para poder difundir conocimientos.

- Los productos de un observatorio pueden servir, al menos, para: a. Caracterizar una situación o momento b. Apoyar la toma de decisiones coyunturales c. Formular escenarios a futuro.

Es decir un Observatorio Turístico fomenta el incremento de información fiable y verificable de forma organizada con el objetivo de advertir hechos sobre una actividad en específico basado en datos reales, ademas un OT basan gran parte de su estudio en el desarrollo de indicadores determinantes para una evaluación adecuada de la oferta y demanda circulante en su mercado.

Con el propósito antes mencionado la necesidad de generación de indicadores constituye un herramienta vital para determinar y medir resultados del turístico, por ende en el modelo de gestión para el OT de la Universidad Estatal Amazónica (UEA) en el año 2018 propone el desarrollo de indicadores y define dentro de su estructura un sección para informes económicos en que se fija un apartado de gasto y visitación.

\section{El turismo y su impacto sobre la economía}

"La importancia económica del turismo es innegable" afirma (Corral, 2017, pág. 63), hecho dado por un conjunto de actividades en las que el turismo se apoya para dar lugar a un producto determinado para la demanda mientras que la oferta procura satisfacer las 
necesidades de los clientes o turistas. Es importante resaltar los hechos económicos mediante los principales indicadores econométricos a través de un breve resumen sobre la situación del país.

El factor económico es determinante para el desarrollo tanto de las empresas como del mercado, en este sentido el análisis de la dimensión económica establece los factores generadores de oportunidades y/o amenazas para el desenvolvimiento de la actividad turística; entre los elementos más importantes para una evaluación económica se tiene a la Inflación, Producto Interno Bruto (PIB), Balanza Comercial y Riesgo País. Por lo tanto, el desarrollo económico del turismo puede partir de la teoría de desarrollo de Fontela y Guzmán (2003) a quien cita Ordóñez (2014, pág. 414) que enfatiza sobre la teoría circular de desarrollo en la cual se lleva a colación tres dimensiones: política, económica y social, como se presenta en la siguiente Figura 1:

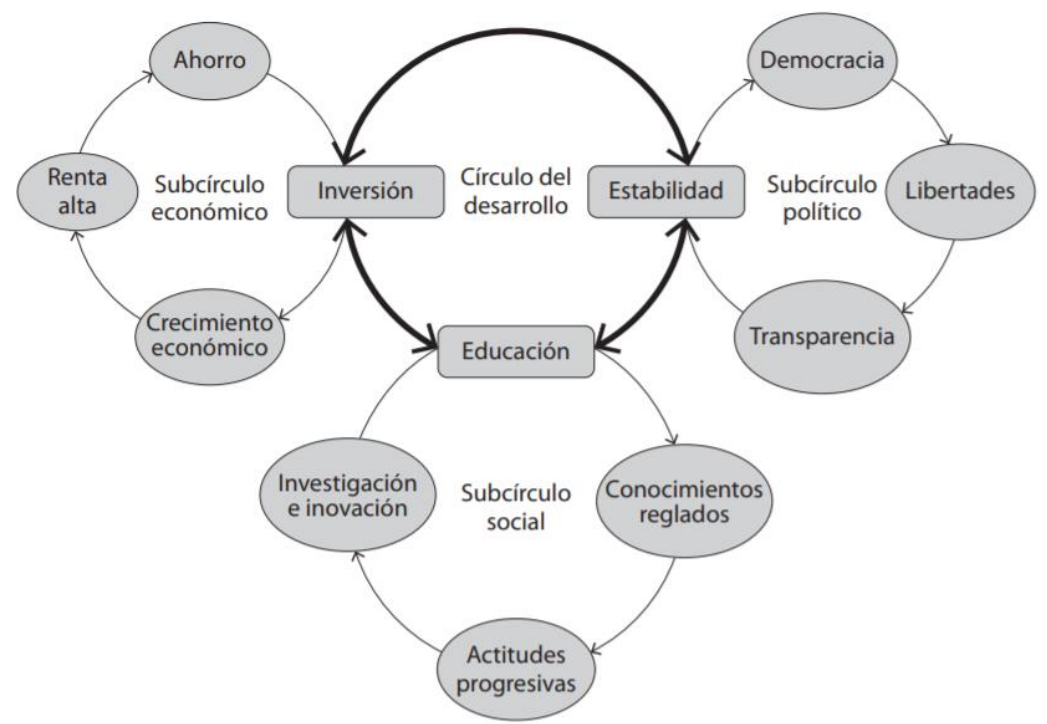

Figura 1. Teoría circular de desarrollo

Fuente: (Fontela y Gusmán, 2003 a quien cita Ordóñez, 2014)

Afirmando lo expuesto en la figura anterior dentro de una teoría circular de desarrollo se estimula la creación de modelos que maximicen la capacidad generar emprendimientos ligados a la generación de empleos y empleabilidad en diferentes sectores económicos, lo cual reafirma que el desarrollo y la economía se encuentran estrechamente entrelazadas fomentando el crecimiento económico, a esto le suceden la estabilidad política y social junto con la facultada progresista de los individuos para investigar e innovar (Villena y Galiano, 2017, pág. 10).

Tal es el caso que el desarrollo económico expresa que entre más contundente sea el factor empresarial su desarrollo económico es creciente en un territorio. "Acorde con lo mismo, el 
desarrollo económico puede definirse genéricamente como crecimiento sostenible desde tres puntos de vista: económico, social y medioambiental" (de Tomas, y otros, 2003, pág. 68).

Continuando con la línea del desarrollo económico se identifica al turismo como un factor de su crecimiento lo cual se reafirma con (Calvo, 2016, pág. 265) "el turismo genera directa o indirectamente un incremento de la actividad económica y no solo una fuerza trasnformadora, también tiene un papel protagonista en la promoción del diálogo intercultural y conservación del patrimonio natural y cultural" es claro que el turismo provee a la economía aportes significativos en función de sus actividades directas e indirectas, para Quintero (2014) el turismo proporciona significativamente al desarrollo económico de tal forma que su impacto produce una cadena impactos económico y se demuestra mediante la Figura 2:

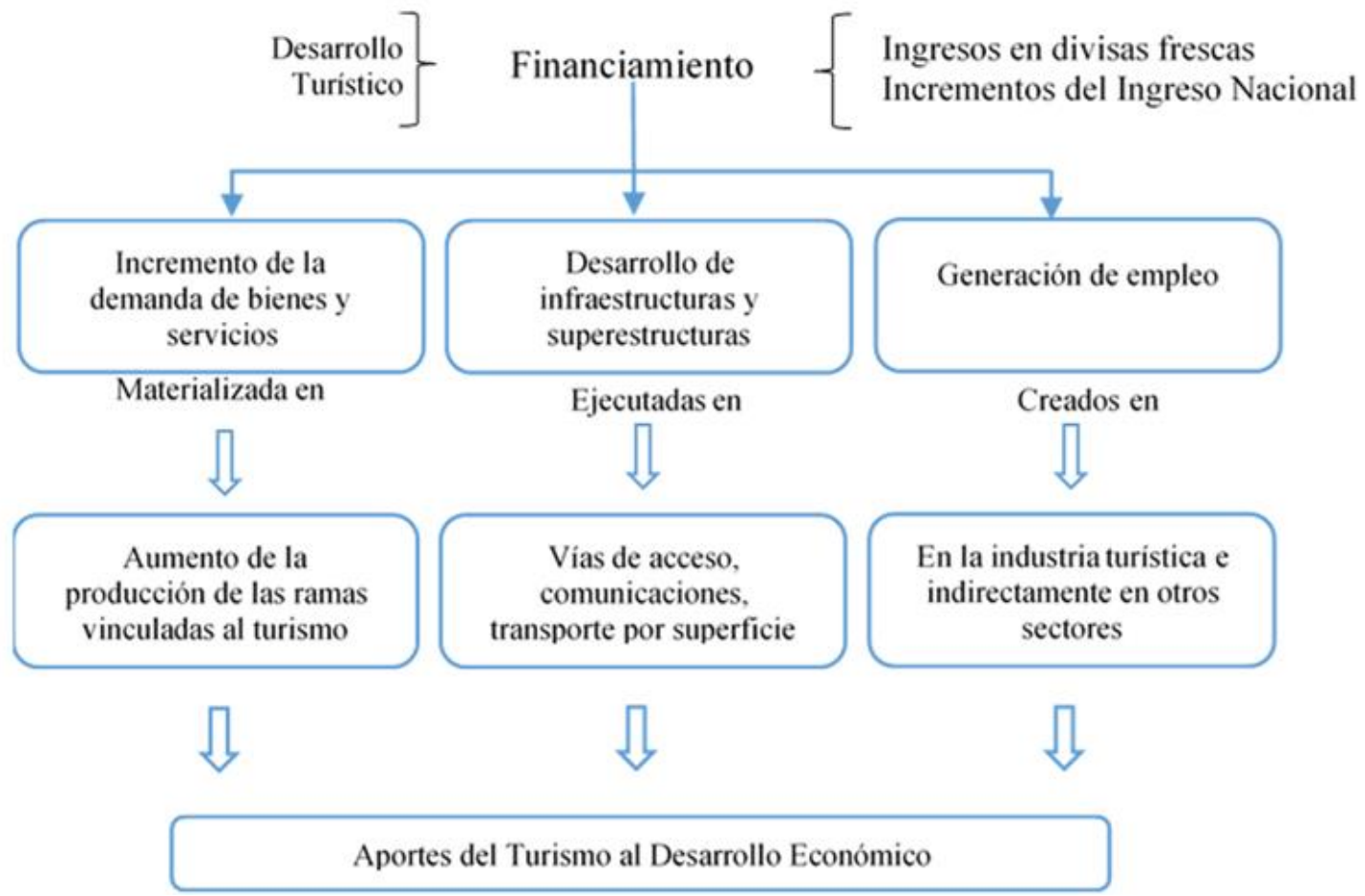

Figura 2. Cadena de impactos económicos del turismo

Fuente: (Quintero, 2004, pág. 263)

En la Figura 2 se demuestra como el turismo impacta significativamente en el desarrollo económico a partir de la creación de productos turísticos generadores potenciales de demanda en bienes y servicios, así como también de infraestructuras y superestructuras permitiendo la dinamización económica mediante inversión que a su vez origina nuevas fuentes de empleo dentro de la actividad turística y las vinculadas a la misma aumentando el ingreso de divisas frescas para su financiamiento.

De la misma forma es imprescindible tomar en cuenta desde el punto de vista turístico como lo define Orozco, Núñez, y Virgen, (2008) "El desarrollo turístico tiene que fundamentarse sobre criterios de sostenibilidad, es decir, ha de ser soportable ecológicamente a largo plazo, 
viable económicamente y equitativo desde una perspectiva ética y social para las comunidades" (pág. 23). Para afianzar los parámetros antes mencionados el desarrollo económico del turismo debe ser equilibrado y se debe apoyar en las condiciones o características que sugiere un verdadero impacto del sistema turístico para la economía.

\section{El sistema turístico}

Por lo anteriormente expuesto sobre el turismo y su impacto en la economía, se hace referencia en el estudio del sistema turístico en el cual se puede identificar el factor económico como parte de su estructura, en base a la capacidad y facilidades que cada lugar posee siendo capaz de adaptarse articuladamente o progresivamente al sistema turístico, con el fin de adoptar estrategias turísticas ideales; es oportuno enfatizar que el aprovechamiento adecuado, racional y respetuoso de los recursos turísticos culturales y naturales que constituyen las bases fundamentales para diversificar la economía local forman parte de las dimensiones expuestas por el autor y se explican mediante la siguiente Figura 3:

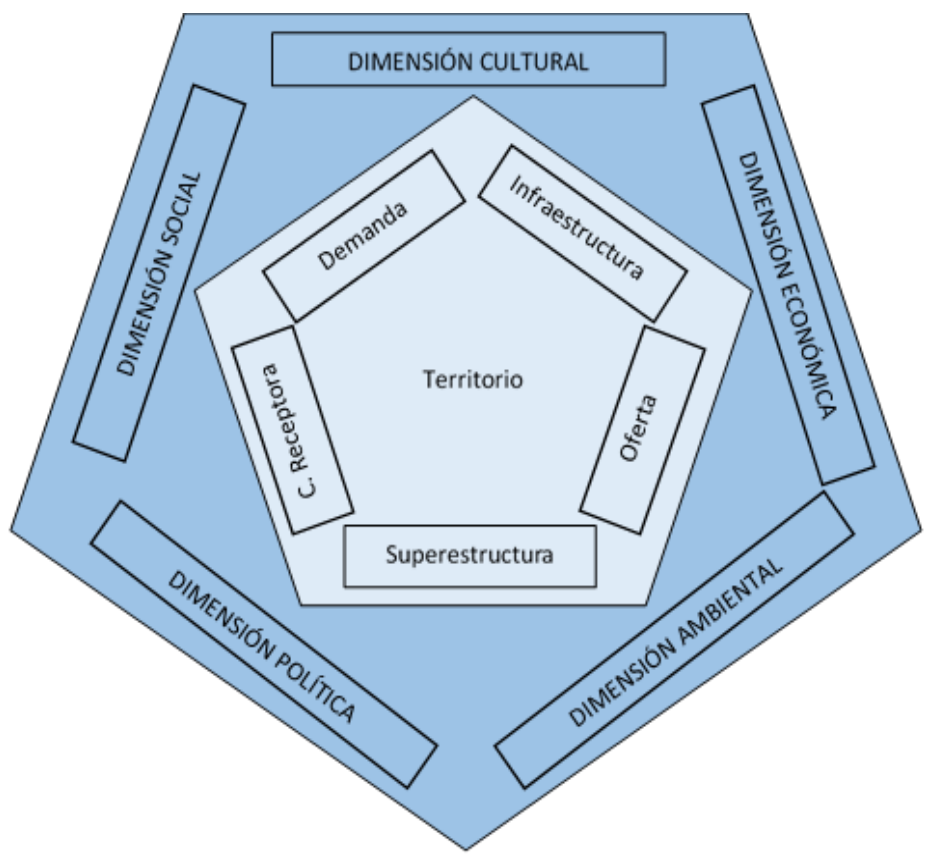

Figura 3. El Sistema Turístico

Fuente: (Varisco, 2013, pág. 66)

De la misma forma Varisco en el año 2013 en su estudio plantea como subsistemas del sistema turístico son la primera etapa de análisis como se demuestra en la siguiente Tabla 1: 


\section{Tabla 1}

Los Subsistemas del Sistema Turístico

Subsistemas Descripción

La demanda turística: Es el número de personas que viajan o desean viajar para utilizar instalaciones turísticas y servicios lejos de sus lugares de trabajo o residencia (Mathieson, 1990). Esta definición contempla la demanda real o efectiva, y la demanda potencial que estaría interesada en viajar al destino. Los estudios de este subsistema contemplan de manera general dos objetivos descriptivos: cuantificar y cualificar la demanda.

En un nivel de análisis más profundo se estudian los determinantes de la demanda, históricos y actuales, y el proceso de decisión de un viaje turístico. También se analizan los cambios en la demanda y la manera en que surgen nuevos segmentos de mercado.

La oferta turística: $\quad$ Es el conjunto de bienes y servicios puestos en el mercado. La oferta se clasifica en primordial y complementaria (De la Torre, 1980): la primera está integrada por los recursos turísticos y la segunda, por las empresas que componen el equipamiento turístico y los eslabonamientos.

La infraestructura: Es el conjunto de obras y servicios que sirven de base para el desarrollo de todas las actividades económicas y, en consecuencia, puede ser de uso común o puede ser específica del turismo. Es el soporte que permite la conexión entre centros emisores y receptores y el desarrollo del destino turístico, en especial, el transporte (rutas y terminales) y la comunicación.

La superestructura: De manera simplificada, suele decirse que el sector público representa al gobierno, el sector privado al mercado y el tercer sector a la sociedad civil. En este subsistema se incluyen las instituciones educativas, de gestión pública o privada, que generan capacitación turística en todos los niveles. También los centros de investigación y desarrollo tecnológico forman parte de la superestructura y en la medida en que la producción de conocimiento esté vinculada a las necesidades humanas, pueden constituirse actores de importancia dentro del sistema.

\begin{tabular}{ll}
\hline La comunidad & La población local, que no participa de manera directa en el resto de los \\
receptora: & subsistemas, tiene fundamental importancia para el desarrollo local. Esta \\
& teoría, en su aplicación al turismo, se ocupa de analizar las condiciones en \\
& que el conjunto de la población puede mejorar su calidad de vida a partir de \\
& la actividad turística.
\end{tabular}

Fuente: (adaptado de Varisco, 2013)

En consecuencia, Varisco sugiere que los subsistemas alineados a las dimensiones facilitan la organización sistemática estructurada de manera específica para el desarrollo de actividades turísticas como se identifica en la siguiente Figura 4: 


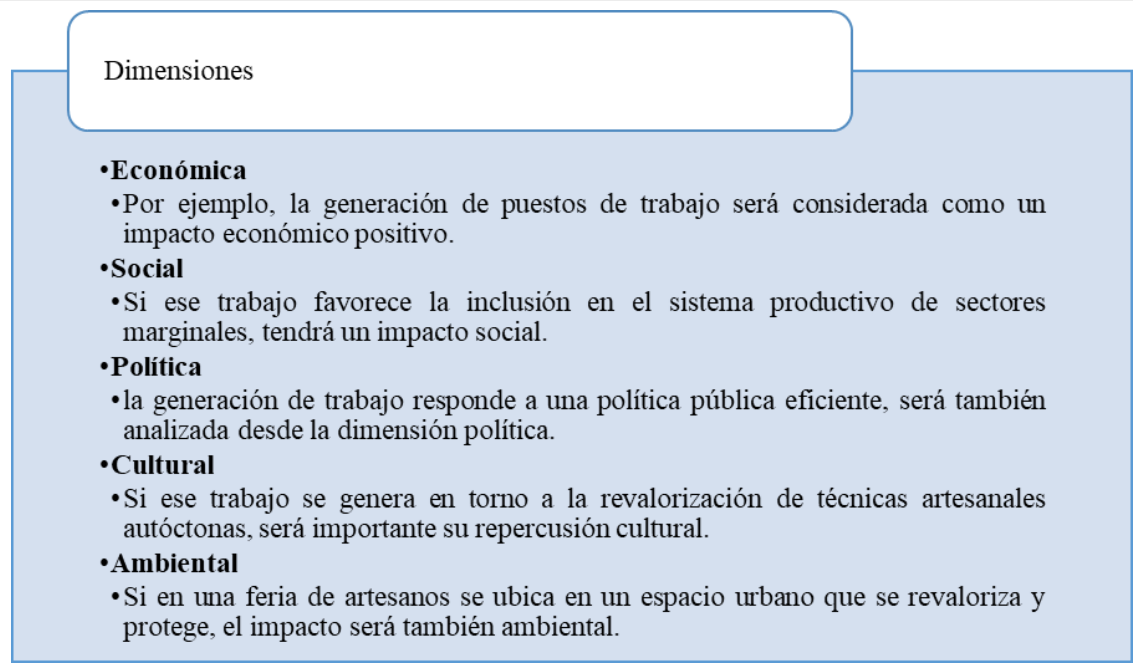

Figura 4. Las Dimensiones del Sistema Turístico

Fuente: (Molina, 2019 quien cita a Varisco, 2013)

Ante lo propuesto anteriormente por el autor es de fundamental trascendencia un pertinente y adecuado enfoque sistémico que integre la división contextual en la que opera el turismo, para definir medidas y herramientas oportunas que defina su propósito en un marco regulador y organizado de manera sistémica.

\section{Observatorio Turístico de la UEA e indicadores económicos básicos de turismo para Pastaza}

Siendo el turismo una actividad dependiente de la economía y viceversa es imprescindible tener mecanismos para evaluar su incidencia en el mercado, por lo cual la OMT máxima entidad de turismo en el mundo basa su desarrollo en el estudio económico del turismo mediante varios recursos y herramienta como barómetros mediante los cuales identifica las tendencias económicas en las que el turismo se desenvuelve, la actividad turística está en su total capacidad para medir su accionar económico.

De acuerdo con Brazales (2018): "la estructura y enfoque de los observatorios depende en gran medida de la sensibilidad del contexto de la sostenibilidad y de las características específicas de los destinos" (pág. 33).

En el Ecuador el MINTUR es el encargado de llevar acabo funciones mediante las cuales se evalúa el factor económico del turismo, es preciso detallar que en el 2011 nace una propuesta de creación de un OT en la Universidad Estatal Amazónica (UEA) y su primordial objetivo fue el de proveer estadísticas pertinentes y confiables que este al alcance de los diferentes usuarios tanto públicos y privados, siendo la información de carácter relevante la cual indique la estructura, comportamiento para brindar una opción frente a la escasa información turística existente en su momento; por tal motivo la UEA junto con su equipo técnico elaboró un 
modelo de gestión del OT-UEA para trabajar con el fin de generar información y divulgación de datos sectoriales basado en criterios académico y científico por medio de ejercicios, ensayos de recolección además de análisis de información secundaria todos ellos relacionados a diversos estudios de turismo con la participación en su formulación, desarrollo y ejecución por parte de los estudiantes y docentes de la carrera de Turismo (UEA, 2018).

El OT-UEA propone en su metodología varias pautas para el desarrollo de sus objetivos una la primera fue la identificación de indicadores de demanda (tradicionales) para Pastaza como se observa en la siguiente Figura:

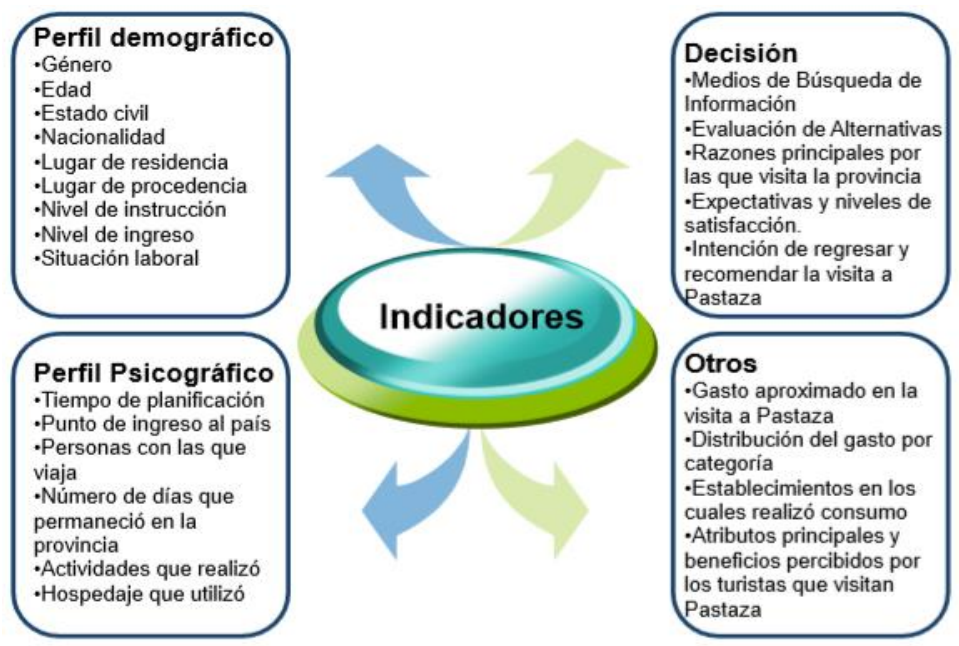

Figura 5. Indicadores de demanda

Fuente: (Universidad Estatal Amazónica, 2011, pág. 90)

Cabe señalar que los indicadores de turismo se enfocan en el desarrollo turístico de la provincia de Pastaza, el OT-UEA como segundo aspecto sugirió la determinar los puntos de recolección de datos o el trabajo de campo junto con un método de levantamiento de información que se muestra a continuación:

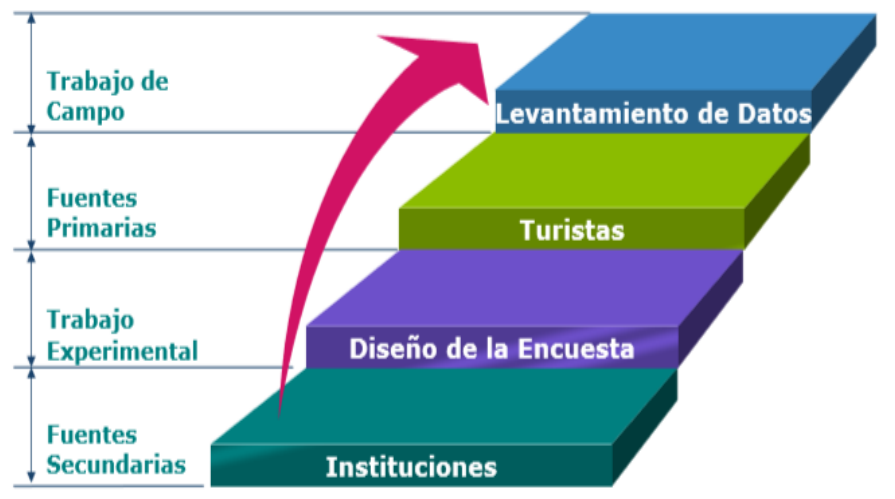

Figura 6. Esquema de investigación de campo

Fuente: (UEA, 2011, pág. 87) 
Y como ultimo procedimiento en su metodología el OT de la UEA indica el procedimiento para aplicar el cálculo mediante muestreo probabilístico.

Por otra parte, es importante señalar que en el Ecuador existen observatorios turísticos de los que destacan:

- Observatorio de turismo de Galápagos: su principal objetivo "es generar información actualizada sobre la dinámica de la actividad turística en las islas, para orientar la toma de decisiones con miras a alcanzar el modelo de ecoturismo en la provincia" (Observatorio de Turismo Galápagos, 2017)

- Observatorio turístico de la Región Sur del Ecuador: su principal objetivo "es facilitar la información necesaria para la toma de decisiones de los diferentes agentes económicos Implicados en el sector turístico, al mismo tiempo que se evalúa el impacto que las políticas públicas e Iniciativas de cualquier otra índole" (Universidad Técnica Particular de Loja, 2017).

- Observatorio de turismo de la Universidad Tecnológica Equinoccial UTE: su fin consiste en “...el intercambio de información entre la Academia, el sector Público y privado, para disponer de una herramienta estadística de recopilación y generación de información sobre esta actividad turística, permitiendo contar con datos sistematizados, ordenados y actualizados" (Universidad Tegnológica Equinoccial, 2016).

De acuerdo con lo expuesto anterior mente en el Ecuador existen observatorios turísticos que destina su labor a la recopilación, desarrollo y generación de información turística mediante indicadores para una adecuada toma de decisiones en el ámbito turístico. Soldevila y Roca, (2004) adapta una metodología para la creación de indicadores y explica las cualidades que deben perseguir para ser funcionales:

- Relevancia: deben ser útiles para la toma de decisiones de los gestores de la actividad a la que se refiere el indicador.

- Verificable: la interpretación de los mismos debe ser la misma para todos los usuarios.

- No sesgados: el calculo de los indicadores no puede tener sesgo estadístico ni personal.

- Cuantificables: se tiene que estimar por medio de cantidades numéricas.

- Coste/eficacia: que el beneficio del uso del indicador sea superior al coste de su calculo e implantación.

- Aceptabilidad: los usuarios de los indicadores deben aceptarlos, previamente a su utilización, como indicadores útiles y correctos para medir la actividad en concreto (pág. 85). 


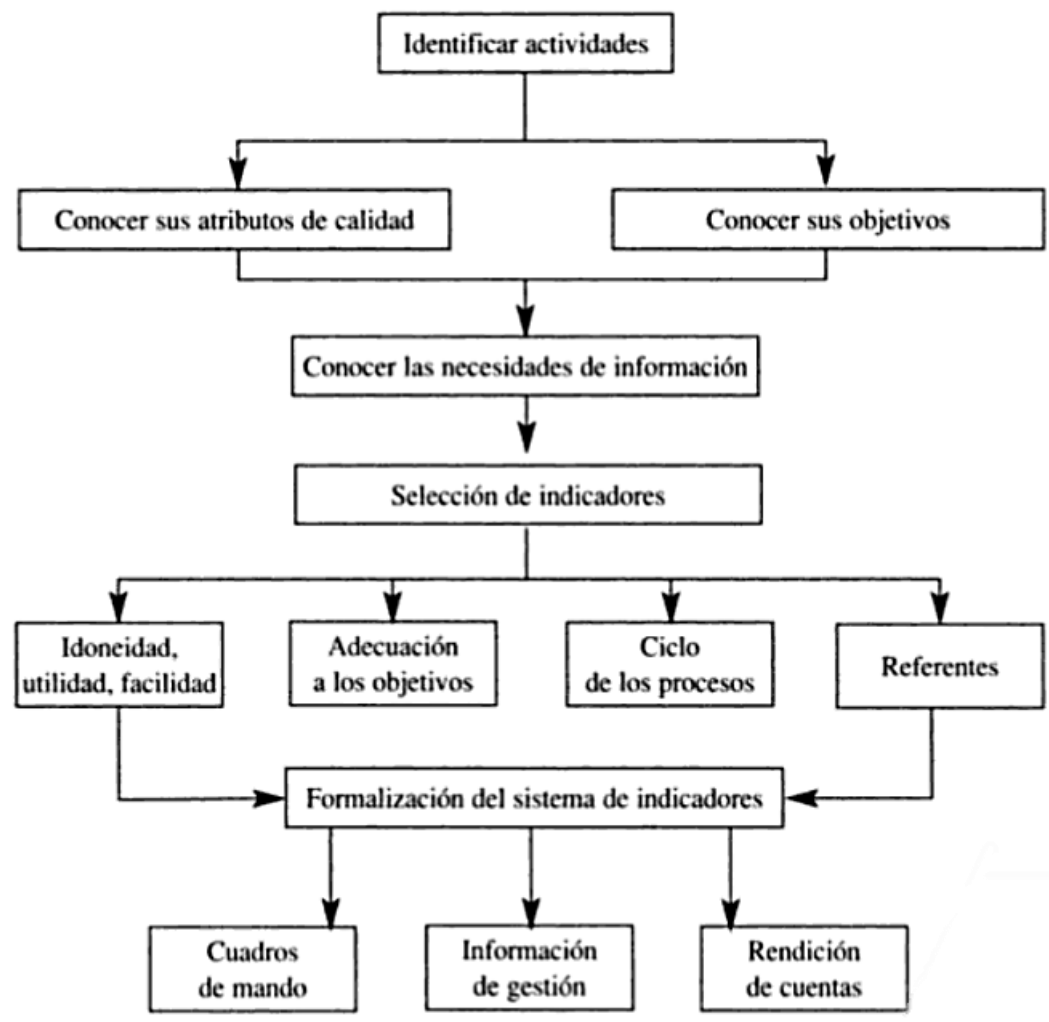

Figura 7. Metodología a seguir para la construcción de indicadores

Fuente: (Soldevila y Roca, 2004, pág. 86 quien cita a AECA 1997, pág. 39; López y Gadea, 1992, pág. 89)

Para Márquez,y otros, (2020) "Los indicadores representan una herramienta fundamental para medir los resultados de la gestión turística en términos de progreso hacia la sostenibilidad" (pág. 3), también detalla el proceso metodológico para la elaboración de indicadores de sostenibilidad económica del sector hotelero desarrollado en fases:

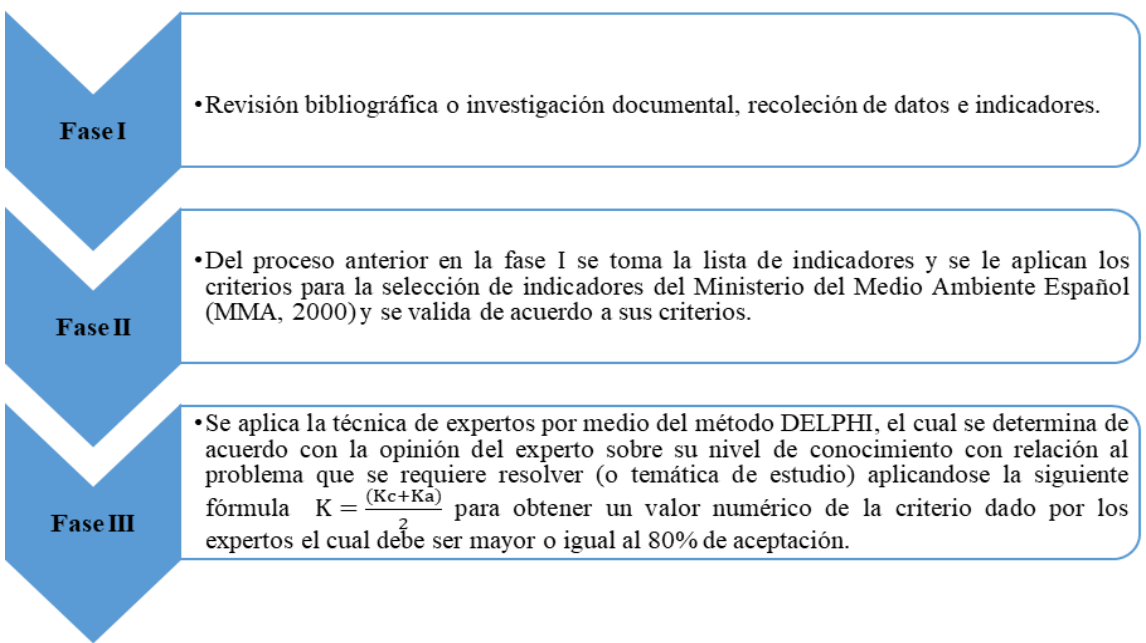

Figura 8. Proceso metodológico para la elaboración de indicadores de sostenibilidad económica del sector hotelero

Fuente: (adaptado de Márquez y otros, 2020) 
De acuerdo con los procesos metodológicos señalados anteriormente para la construcción y definición de indicadores los autores muestran la identificación de necesidades y la recolección de datos con la finalidad de conocer las necesidades y actividades que se desarrolla en un lugar para en función de ello establecer los indicadores a usar o crear, para tener una óptica mas amplia de lo antes señalado Castillo, Polo y Uruchima, (2018) en su articulo determinan que los indicadores de económicos constituyen un medio objetivo en la medición de la incidencia turística y exponen algunos indicadores y su función los que permiten abordar parámetros sobre el turismo tales como el nivel de producción turística, la renta turística y su relación con el PIB, indicador de competitividad de la demanda turística, Multiplicador de la producción turística sobre el resto del sistema económico entre otros (pág. 156).

Tabla 2

Indicadores de naturaleza económica para medir el impacto del turismo a nivel local

\begin{tabular}{|c|c|}
\hline Indicador & Función que cumple \\
\hline $\begin{array}{l}\text { Nivel de producción turística } \\
\text { directa (PTD) }\end{array}$ & $\begin{array}{l}\text { Mide la evolución de las ventas que las empresas y sectores, } \\
\text { directamente relacionadas y relacionados con el gasto de los } \\
\text { turistas realizan. }\end{array}$ \\
\hline $\begin{array}{l}\text { Renta Turística y su relación con } \\
\text { el PIB local o regional. Según } \\
\text { períodos anuales o trimestrales y } \\
\text { su comparación temporal } \\
\text { (RTRL) }\end{array}$ & $\begin{array}{l}\text { Valora la parte de la producción turística que se transmite como } \\
\text { renta o valores añadidos al Producto Interior Bruto del país. Es un } \\
\text { indicador básico, puesto que resume el conjunto de la aportación } \\
\text { económica del turismo en el marco del área geográfica que se } \\
\text { desee conocer y proyectar políticamente. }\end{array}$ \\
\hline $\begin{array}{l}\text { Indicador de competitividad de } \\
\text { la demanda turística }\end{array}$ & $\begin{array}{l}\text { Establece por medio de un indicador cuál es el grado o escala de } \\
\text { competitividad de los diferentes municipios, permitiendo una } \\
\text { comparación homogénea }\end{array}$ \\
\hline
\end{tabular}

Multiplicador de la producción turística sobre el resto del sistema económico

Permite valorar los efectos indirectos e inducidos causados por el gasto de los turistas, de manera que puede tenerse una percepción global de los efectos del turismo

Valoración del esfuerzo
presupuestario municipal en la
promoción de la demanda
turística

Correlación anual o dependencia del turismo en el destino, respecto al conjunto del turismo en el país (residentes y no residentes)

Indicador de dependencia del crecimiento en los destinos turísticos en función de la variación de la renta de los países emisores

\begin{tabular}{|c|c|}
\hline $\begin{array}{l}\text { Valoración en el ámbito } \\
\text { municipal de indicadores de } \\
\text { productividad de empleo e } \\
\text { inversión hotelera en los destinos } \\
\text { turísticos }\end{array}$ & $\begin{array}{l}\text { La eficacia alcanzada por los factores productivos es una } \\
\text { magnitud necesaria si se pretende mejorar en cuanto a los } \\
\text { resultados parciales y generales del proceso de producción }\end{array}$ \\
\hline
\end{tabular}

Fuente: (Castillo, Polo y Uruchima, 2018 quienes citan a Figuerola y cols., 2015)

La comparación de la acción presupuestaria municipal, y la valoración conseguida de los efectos e influencia del turismo, medirá la eficiencia de la acción política, respecto al presupuesto determinando la validez de la cantidad, así como la distribución de las partidas.

El indicador establece en un cuadro comparativo, como los comportamientos de la estrategia turística ha tenido lugar a nivel del municipio, y nivel nacional, manifestando el grado de dependencia posible, entre el conjunto total, y cada una de sus partes

Es conveniente disponer de un instrumento de previsión, que permita indicar o alertar, como los comportamientos de las demandas turísticas generales, pue- den influir en el marco local. 
En otra instancia la Comisión Económica para América Latina y el Caribe (CEPAL) en el año 2006 propone una metodología para el cálculo de indicadores económicos básicos a usarse en el análisis turístico agrupándolos en 4 grupos:

- Indicadores de Equilibrio macroeconómico

- Producción

- Consumo

- Empleo y salarios

\section{Metodología}

Para el desarrollo metodológico de la investigación se parte de un estudio cualitativo marcando relación en el enfoque positivista, la cual es de carácter comprensivo y constructivista, identificando principalmente características determinadas por las bases teóricas, sobre el impacto económico del turismo, el sistema turístico y observatorios turísticos e indicadores junto con sus formas de medir. A través de un alcance exploratorio para descubrir y prefigurar sobre información documentada de indicadores económicos de turismo para la provincia de Pastaza y su enfoque al OT-UEA

Mientras que para el desarrollo de la investigación se determinó el uso de un método documental Para el desarrollo del estudio se utilizó una perspectiva teórica "que consiste en sustentar teóricamente el estudio, una vez que ya se ha planteado el problema de investigación" (Hernández, Fernández, y Baptista, 2014, pág. 60). De mismo modo se aplicó de análisis histórico lógico que triangula y permite una estructura lógica de información documental a partir de análisis:

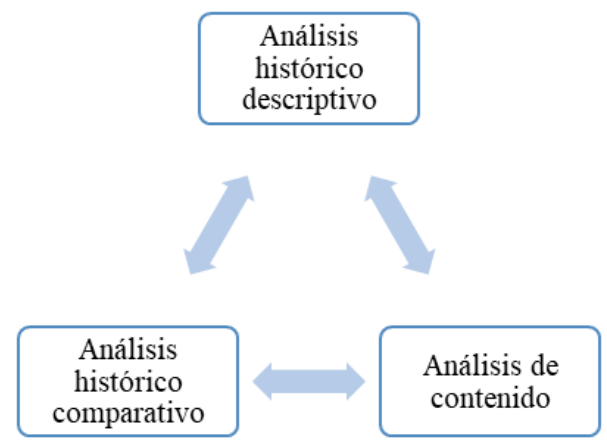

Figura 9. Triangulación de estructura lógica de análisis

Fuente: (Figueredo, 2020, pág. 51)

Por otra parte junto la figura anterior la investigación se apoya para el desarrollo del Análisis de Indicadores económicos de Turismo para la provincia de Pastaza enfocados al Observatorio Turístico "UEA" basado en lo expuesto por Arias 2012 a quien cita Chávez y 
otros, (2016) quien menciona que el diseño de la investigación se clasifica: en documental, de campo y experimental; y este caso de estudio se inclina en el ámbito documental de lo cual expresa el autor antes mencionado como un soporte de material documentado para su desarrollo.

\section{Resultados}

Dado que la investigación es de tipo documental y con un enfoque cualitativo, se determina los indicadores basados en estudios presentes y pasados para llegar a un consenso y evidenciar su efectividad siendo estos viables, verificables, cuantificables, que su desarrollo sea eficiente en función de su aplicación en cuanto al costo que este incurra se determine su beneficio en mayor medida que su gasto de tal forma que su utilidad permite la toma de decisiones acertadas en materia turística.

\section{Tabla 3}

Indicadores económicos de turismo

\begin{tabular}{|c|c|c|c|c|c|}
\hline $\mathbf{N}^{\circ}$ & Indicador & Fórmula de cálculo & Unidad de medida & $\begin{array}{l}\text { Periodicidad } \\
\text { de cálculo }\end{array}$ & $\begin{array}{l}\text { Tendencia } \\
\text { deseable }\end{array}$ \\
\hline 1 & $\begin{array}{l}\text { Ingreso } \\
\text { promedio } \\
\text { por } \\
\text { habitaciones } \\
\text { disponibles }\end{array}$ & $\begin{array}{l}\text { Ingreso total/Número total } \\
\text { de habitaciones disponibles }\end{array}$ & $\begin{array}{l}\text { Se expresa en } \\
\text { monetario } \\
\text { habitación. }\end{array}$ & Anual & Creciente \\
\hline 2 & $\begin{array}{l}\text { Índice de } \\
\text { ingresos y } \\
\text { gastos por el } \\
\text { servicio } \\
\text { hotelero }\end{array}$ & $\begin{array}{l}\text { (Valor monetario de los } \\
\text { ingresos y gastos por } \\
\text { concepto } \\
\text { alojamiento/Total de } \\
\text { ingresos y gastos de la } \\
\text { instalación hotelera) } * 100\end{array}$ & $\begin{array}{l}\text { Porcentaje del índice } \\
\text { de ingresos y gastos } \\
\text { por el servicio } \\
\text { hotelero }\end{array}$ & Anual & Creciente \\
\hline 3 & $\begin{array}{l}\text { Impacto } \\
\text { económico } \\
\text { del servicio } \\
\text { hotelero en } \\
\text { la localidad }\end{array}$ & $\begin{array}{l}\text { (Número de directivos y } \\
\text { clientes } \\
\text { satisfechos con el resultado } \\
\text { obtenido de este } \\
\text { indicador/Total } \\
\text { directivos y de } \\
\text { internos en la instalación } \\
\text { hotelera) } * 100\end{array}$ & $\begin{array}{l}\text { Valoración } \\
\text { cualitativa realizada } \\
\text { por los directivos y } \\
\text { clientes internos de la } \\
\text { instalación hotelera } \\
\text { sobre el cumplimiento } \\
\text { de las } \\
\text { normas, políticas, } \\
\text { resoluciones, leyes y } \\
\text { estrategias } \\
\text { ambientales. }\end{array}$ & Anual & Creciente \\
\hline 4 & $\begin{array}{l}\text { Índice de } \\
\text { consumo de } \\
\text { agua por } \\
\text { habitaciones } \\
\text { días } \\
\text { ocupadas }\end{array}$ & $\begin{array}{l}\text { (Consumo en litros } \\
\text { mensual de agua (L/mes) } \\
\text { /Número de pernoctaciones } \\
\text { en un mes (pax/mes) por } \\
\text { habitaciones ocupadas) } \\
* 100\end{array}$ & $\begin{array}{l}\text { Valor porcentual del } \\
\text { índice de consumo de } \\
\text { agua por habitaciones } \\
\text { ocupadas en el mes. }\end{array}$ & Mensual & Decreciente \\
\hline 5 & $\begin{array}{l}\text { Índice de } \\
\text { consumo de } \\
\text { energía por } \\
\text { habitaciones }\end{array}$ & $\begin{array}{l}\text { (Consumo de kilowatts } \\
\text { hora mensual /Número de } \\
\text { pernoctaciones en un mes } \\
\text { (pax/mes) por habitaciones } \\
\text { ocupadas) *100 }\end{array}$ & $\begin{array}{l}\text { Valor porcentual del } \\
\text { índice de consumo de } \\
\text { energía por } \\
\text { habitaciones } \\
\text { ocupadas en el mes. }\end{array}$ & Mensual & Decreciente \\
\hline
\end{tabular}


días

ocupadas

Fuente: (Márquez, y otros, 2020)

Los indicadores de la tabla anterior se ajustan a la realidad de la provincia de Pastaza aplicada, además del siguiente KPI (key performance indicator) dentro del área o industria hotelera, también puede posibilitar la toma de datos mediante el índice RevPar en el cual se expresa la tarifa promedio por habitación disponible y la fórmula es $R E V P A R=O R \times A R R$ donde $O R$ es el resultado del número de habitaciones vendidas por el número de habitaciones disponibles por 100 y su resultado se expresa en porcentaje mientras que $A R R$ es el resultado de ingreso por alojamiento sobre el número de habitaciones vendidas, este índice sirve para comparar establecimientos de alojamiento de alto radio de ocupación y su tarifa promedio con hoteles de baja ocupación y alta tarifa promedio (Centeno, 2014, pág. 25).

Es imprescindible señalar que los indicadores económicos de turismo para un lugar en específico deben planearse en función de sus necesidades y la toma de datos depende de la misma primicia ya que no sería eficiente la toma información, para lo cual se sugiere identificar el desarrollo local y las características que posea para obtener resultados útiles.

Las metodologías expuestas para los casos de desarrollo determinan la posición de diversos autores mediante análisis de los cuales arroja la función de los indicadores en concordancia con el impacto económico que desarrolla el turismo en la economía y el OT-UEA con las bases de datos de información primaria, teóricas e históricas además de la OMT y el MINTUR en los cuales se refieren a las características que el turismo posee sobre la medición económica por medio de indicadores económicos turísticos junto con la afirmación y desarrollo de investigaciones de diversos autores; del mismo modo la presentación de metodologías para el desarrollo de indicadores y su proceso de construcción.

Es importante indicar que para definir y analizar el sector turístico se requiere primero evaluar la importancia del turismo en la economía del país, en términos de la oferta, demanda, generación del capital, la contribución al PIB con modelos diferenciadores adaptados al turismo y no a la economía como tal partiendo de las características circundantes al turismo orientadas a las demandas actuales. Es fundamental señalar que, el indicador PIB dimensiona la notable importancia del turismo para con la economía que puede ser medida en el país y debería desarrollarse su cálculo en la región de estudio bajo parámetros de comparación, de acuerdo con lo expuesto por las Naciones Unidas en el 2010 en su página 50 en la Cuenta Satélite de Turismo: Recomendaciones sobre el Marco Conceptual a quien cita el (Instituto Nacional de Estadísticas y Censos, 2016, pág. 1) se indica la contribución del PIB al turismo y la función del indicador con su fórmula: 
La parte del PIB atribuible directamente al consumo turístico interior puede medirse como la suma del Valor Añadido Bruto (a precios básicos), generado por todas las industrias en respuesta al consumo turístico interior más los impuestos netos sobre los productos e importaciones, incluidos en el valor de este gasto a precios de adquisición.

Tabla 4

Producto Interno Bruto Directo Turístico

\begin{tabular}{|c|c|c|c|c|}
\hline Indicador & Fórmula de cálculo & $\begin{array}{l}\text { Unidad de } \\
\text { medida }\end{array}$ & Interpretación & $\begin{array}{c}\text { Periodicidad de } \\
\text { cálculo }\end{array}$ \\
\hline $\begin{array}{l}\text { Producto Interno } \\
\text { Bruto Directo } \\
\text { Turístico } \\
\text { (PIBDT) }\end{array}$ & $\begin{array}{l}\mathrm{PIBDTPt}=(\mathrm{PIBDTPt} / \mathrm{P} \\
\mathrm{IBt}) * 100\end{array}$ & $\begin{array}{l}\text { Se expresa en } \\
\text { porcentaje }\end{array}$ & $\begin{array}{l}\text { Para el año t, el } \\
\text { PIBDT aportó } \\
\text { con alrededor } \\
\text { del XX\% al PIB } \\
\text { nacional. }\end{array}$ & Anual \\
\hline
\end{tabular}

Fuente: (Instituto Nacional de Estadísticas y Censos, 2016, pág. 2)

Tabla 5

Número de establecimientos productivos por cantones de la Provincia de Pastaza

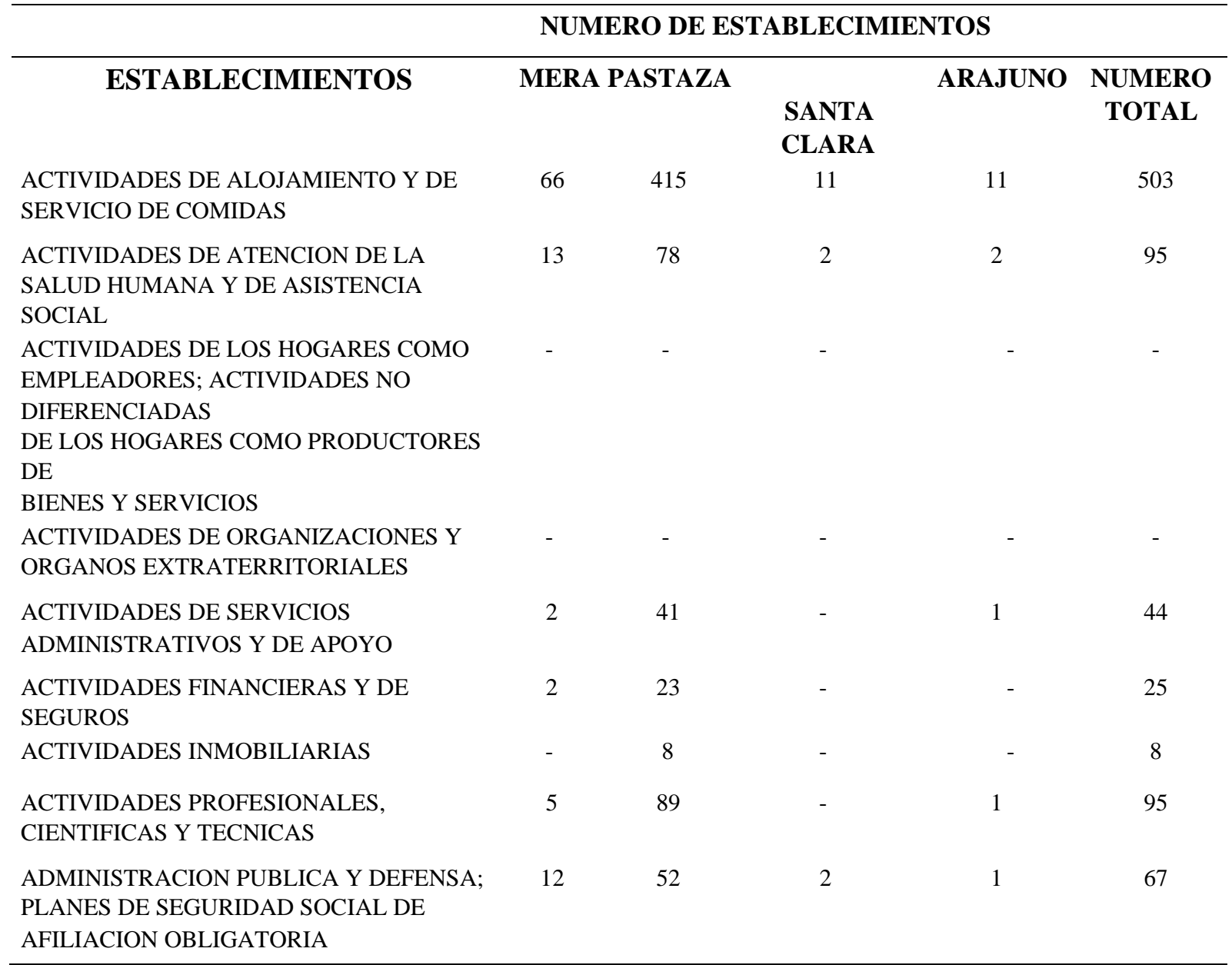




\begin{tabular}{|c|c|c|c|c|c|}
\hline $\begin{array}{l}\text { AGRICULTURA, } \\
\text { SILVICULTURA Y PESCA }\end{array}$ & - & 3 & - & - & 3 \\
\hline $\begin{array}{l}\text { ARTES, ENTRETENIMIENTO Y } \\
\text { RECREACION }\end{array}$ & 11 & 32 & 1 & - & 44 \\
\hline $\begin{array}{l}\text { COMERCIO AL POR MAYOR Y AL POR } \\
\text { MENOR; REPARACION DE VEHICULOS } \\
\text { AUTOMOTORES Y MOTOCICLETAS }\end{array}$ & 181 & 1.482 & 37 & 23 & 1.723 \\
\hline CONSTRUCCION & 2 & 8 & - & - & 10 \\
\hline $\begin{array}{l}\text { DISTRIBUCION } \quad \text { DE } \\
\text { ALCANTARILLADO, } \\
\text { GESTION DE DESECHOS Y ACTIVIDADES } \\
\text { DE } \\
\text { SANEAMIENTO }\end{array}$ & - & 1 & - & - & 1 \\
\hline ENSEÑANZA & 20 & 84 & 5 & 5 & 114 \\
\hline EXPLOTACION DE MINAS Y CANTERAS & - & - & - & - & - \\
\hline INDUSTRIAS MANUFACTURERAS & 31 & 265 & 2 & 7 & 305 \\
\hline INFORMACION Y COMUNICACION & 26 & 139 & 2 & 1 & 168 \\
\hline OTRAS ACTIVIDADES DE SERVICIOS & 43 & 232 & 4 & 3 & 282 \\
\hline $\begin{array}{l}\text { SUMINISTRO DE ELECTRICIDAD, GAS, } \\
\text { VAPOR Y AIRE ACONDICIONADO }\end{array}$ & - & 2 & - & - & 2 \\
\hline TRANSPORTE Y ALMACENAMIENTO & 13 & 38 & - & - & 51 \\
\hline TOTALES & 427 & 2.992 & 66 & 55 & 3.540 \\
\hline
\end{tabular}

Fuente: (INEC, 2010)

\section{Discusión}

El turismo es un determinante sustancial en el desarrollo económico, sin el desarrollo turístico las diversas herramientas empleadas por la economía mostrarían un resultado negativo en su análisis, el impacto que el turismo produce en la economía fija la necesidad para crear fórmulas de medida que establezcan su incidencia y para valorar idóneamente es necesario definir al sistema turístico como lo plantea Varisco en el año 2014 con dimensiones y subsistemas, que permite de forma sistemática reconocer la fuga de ingresos generados por la actividad turística refiriéndose con un ejemplo; "En algunos casos pueden llegar al 90\% del gasto turístico, es decir, que de cada 100 dólares que se gasta el turista en sus vacaciones, solamente 10 llegan a la economía local" cómo lo afirma el (Fondo Social Europeo; Gobierno de España, 2019, pág. 6).

En cuanto a los indicadores se tiene como resultado que constituyen en un instrumento para medir, mediante un soporte de material documentado para el desarrollo de la investigación lo cual evidencio una connotación variante en función de la operatividad que puede tener cada indicador partiendo de estudios aplicados que desarrollada el OT-UEA, como el perfil del turista que vista el Puyo -Pastaza. 
Por otra parte, el gasto aproximado en visita y los establecimientos en los cuales se realizó el consumo son indicadores susceptibles a una fuga de ingresos locales además de una aproximación no real al gasto que un turista puede en realidad dejar en un destino mientras que el consumo definido en establecimientos está sujeto a parámetros de valoración muy amplios si se especifica un sistema turístico del cual se desea tener datos que permitan tomar decisiones, puesto que, posibilita un sesgo en los datos ya que en mucho de los casos no son solo los turistas quienes consumen en los establecimientos.

De acuerdo con la información recabada y facilitada por el OT-UEA se pudo determinar datos de trascendental importancia que muestra como el turismo se desarrolla en la provincia de Pastaza tales como: llegadas internacionales de acuerdo a la nacionalidad, la motivación de viaje, tasa y tarifas en alojamiento, empleo y empleabilidad en la industria.

\section{Conclusiones}

- El turismo como dinamizador económico promueve la capacidad de medición en diversos ámbitos por lo tanto se requiere crear instrumentos que faciliten su cuantificación a través de contexturas ágiles para obtener resultados eficientes en el menor tiempo posible y con el menor desgaste de recursos.

- El impacto económico que tiene el turismo genera la imperiosa necesidad de evaluar el mercado turístico para obtener información relevante acorde a las necesidades de la oferta y demanda por medio de indicadores, siendo pertinente identificar las alternativas que promueve la actividad mediante información determinada por estructuras turísticas organizadas.

- Las alternativas que ofrecen los observatorios turísticos consisten en ser herramientas estratégicas para valoración científica, permitiendo recopilar información con el fin de generar estadísticas pertinentes y necesarias para el sector turístico en función del tratamiento de datos y su adecuada difusión.

\section{Referencias Bibliográficas}

Angulo, N. (2009). ¿Qué son los observatorios y cuáles son sus funciones? Innovación Educativa, 5-17.

Banco Central del Ecuador. (2 de Julio de 2019). Banco Central del Ecuador. Recuperado el 15 de Septiembre de 2020, de Banco Central del Ecuador Web Site: https://www.bce.fin.ec/index.php/boletines-de-prensa-archivo/item/1182-laeconom\%C3\%ADa-ecuatoriana-creci\%C3\%B3-06-en-el-primer-trimestre-de-2019

Brazales, D. (2018). Indicadores para un Observatorio Turístico Enfocado en las provincias de la zona 3 del Ecuador. Editorial Academia Español.

Calvo, A. (2016). Economía internacional y organismos económicos internacionales (2da Edición ed.). Madrid: Editorial Universitaria Ramón Areces (UNED). 
Castillo, Y., Polo, M., \& Uruchima, F. (2018). El impacto del turismo en la economía local. Una necesidad de medir en el Ecuador. Revista Killkana Sociales. Vol. 2, No. 3, 153160.

Centeno, V. (2014). UF0079 - Gestión de sistemas de distribución global. España: Editorial ELEARNING.

Chávez Meléndez, J., Olguín Murrieta, J. A., González Durán, N. H., Guzmán Obando, J., Garciá Guzmán, J. C., \& Vázquez Pimienta, J. M. (2016). Ciudades Emergentes: Aplicación De Metodología Ices Del Bid En La Zona Sur de Tamaulipas. Palibrio.

Corral, A. (2017). La liberalización del sector turístico: ¿Hacia un modelo de turismo sostenible? Madrid: REUS Editorial.

de Tomas, S., Vaquero, E., \& Valle, J. (2003). El día de Europa: presente y futuro de la Unión Europea. Madrid: Universidad Pontificia Comillas.

Figueredo, E. (2020). Nuevos enfoques de la metodología de la investigación histórica aplicada al deporte. Cuba: Editorial Universitaria.

Fondo Social Europeo; Gobierno de España. (28 de Febrero de 2019). Vinos de Ucles. Recuperado el 2 de Septiembre de 2020, de http://www.vinosdeucles.es/sites/default/files/inlinefiles/4_LIBRO\%20Turismo\%20y\%20Desarrollo.pdf

Heredia, J. (2000). Sistema de indicadores para la mejora y el control integrado de la calidad de los procesos. Universitat Jaume I.

Hernández Sampieri, R., Fernández Collado, C., \& Baptista Lucio, M. d. (2014). Metodología de la Investigación (Sexta Edición ed.). México: MCGRAW-HILL.

Instituto Nacional de Estadísticas y Censos. (2016 de Agosto de 2016). Instituto Nacional de Estadísticas y Censos. Recuperado el 4 de Septiembre de 2020, de Instituto Nacional de Estadísticas $y$ Censos Sitio Web: https://www.ecuadorencifras.gob.ec/documentos/webinec/Sistema_Estadistico_Nacional/Objetivos_Desarrollo_Sostenible_ODS/Objetiv o_8/Meta_8.9/Indicador_8.9.1/FM_PIB_TURISMO.pdf

MÁrqueZ, L., CUÉTARA, L., BERNARDO, J., \& MERA, D. (2020). Sistema de indicadores para la evaluación de la sostenibilidad económica del sector hotelero en la parroquia Crucita, Manabí, Ecuador. Revista ESPACIOS, 3-13.

Ministerio de Turismo (MINTUR). (23 de Diciembre de 2019). Promedio de llegada de visitantes extranjeros a Ecuador creció 4\% en 2019. Quito, Pichincha, Ecuador. 
Recuperado el 4 de Septimbre de 2020, de https://www.turismo.gob.ec/promedio-dellegada-de-visitantes-extranjeros-a-ecuador-crecio-4-en-2019/

Molina, M. (21 de Enero de 2019). Repositorio Dspace ESPE-L. Recuperado el 6 de Septiembre de 2020, de Repositorio Dspace ESPE-L Sitio Web: http://repositorio.espe.edu.ec/xmlui/browse?value=Molina+Enr\%C3\%ADquez\%2C $+\mathrm{Mar} \% \mathrm{C} 3 \% \mathrm{ADa}+\mathrm{Isabel} \&$ type=author

Observatorio de Turismo Galápagos. (Enero de 2017). Observatorio de Turismo Galápagos. Recuperado el 10 de Septiembre de 2020, de Observatorio de Turismo Galápagos Sitio Web: https://www.observatoriogalapagos.gob.ec/

Ordóñez, J. (2014). Teorías del desarrollo y el papel del Estado Desarrollo humano y bienestar, propuesta de un indicador complementario al Índice de Desarrollo Humano en México. Política y gobierno, 21(2), 409-441.

Organización Mundial del Turismo. (Diciembre de 2019). Organización Mundial del Turismo (UNWTO). Recuperado el 12 de Septiembre de 2020, de https://www.unwto.org/unwto-tourism-dashboard

Organización Mundial del Turismo. (6 de Marzo de 2020). ONWTO. Recuperado el 30 de Julio de 2020, de Organización Mundial del Turismo (ONWTO): https://www.unwto.org/es/news/covid-19-la-omt-pide-que-el-turismo-se-incluya-enlos-planes-de-recuperacion

Organizacion Mundial del Turismo. (15 de Septiembre de 2020). Organizacion Mundial del Turismo. Recuperado el 17 de Septiembre de 2020, de Organizacion Mundial del Turismo Página Web: https://www.unwto.org/es/news/las-cifras-de-turistasinternacionales-caen-un-65-en-la-primera-mitad-de-2020-informa-la-omt

Orozco, J., Núñez, P., \& Virgen, C. (2008). Desarrollo Turístico y Sustentabilidad Social. México: Universidad de Guadalajara.

Quintero, J. (2004). Los impactos económicos, socioculturales y medioambientales del turismo y sus vínculos con el turismo sostenible. Revista Anales del Museo de América. No. 12. , 263 -274.

Soldevila, P., \& Roca, E. (2004). La contabilidad de gestión en las organizaciones sin ánimo de lucro. Madrid: Universidad Pontíficia Comillas.

Universidad Estatal Amazónica. (2011). Proyecto De Investigación Observatorio Turístico De La Uea, Como Espacio Que Genera Información Actualizada Y Permanente De La Dinámica De La Actividad Del Turismo, Para La Toma De Desición A Nivel De La Región Amazónica. Puyo: Uea. 
Universidad Estatal Amazónica. (2018). Producto1. Modelo de Gestión del Observatorio Turístico. Puyo: UEA.

Universidad Técnica Particulas de Loja (UTPL). (21 de Julio de 2017). UTPL Observatorio Turístico Región Sur del Ecuador. Recuperado el 5 de Agosto de 2020, de https://observatorioturistico.utpl.edu.ec/

Universidad Tegnológica Equinoccial. (30 de Marzo de 2016). Observatorio de Turismo UTE Ecuador. Recuperado el 10 de Agosto de 2020, de https://www.ute.edu.ec/facultad-de-ciencias-gastronomicas-y-turismo/observatoriode-turismo/

Villena, F., \& Galiano, A. (2017). El Desarrollo Urbano Sostenible Y Sus Implicaciones Para Las Empresas Y Los Territorios. Revista de Estudios Empresariales. Segunda época, 5-15. doi:10.17561/ree.v0i1.3185

Vogeler, C., \& Hernández, E. (2018). Introducción al turismo: Análisis y estructura. Madrid: Ramón Areces Editorial Universitaria.

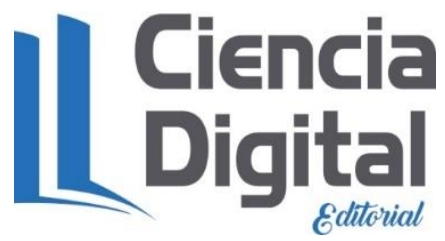




\section{PARA CITAR EL ARTÍCULO INDEXADO.}

Hernández Espinosa, C. del P., Cevallos Punguil, T. C., Cifuentes Caiza, J. A., \& Guerrero Escalante, O. C. (2020). Análisis de indicadores económicos de turismo para la provincia de Pastaza enfocados al observatorio turístico "UEA". Explorador Digital, 4(4), 107-128. https://doi.org/10.33262/exploradordigital.v4i4.1439

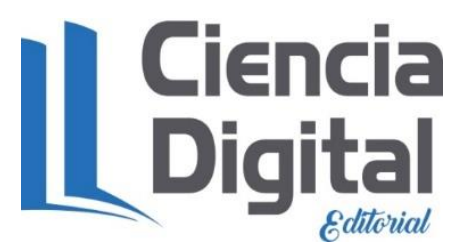

El artículo que se publica es de exclusiva responsabilidad de los autores y no necesariamente reflejan el pensamiento de la Revista Explorador Digital.

El artículo queda en propiedad de la revista y, por tanto, su publicación parcial y/o total en otro medio tiene que ser autorizado por el director de la Revista Explorador Digital.
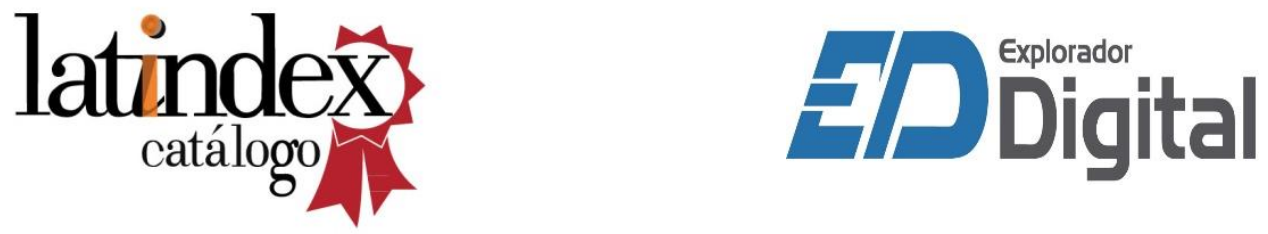